\title{
A Porous Bulk Metallic Glass with Unidirectional Opening Pores
}

\author{
X. H. Chen, ${ }^{a}$ Y. Zhang, ${ }^{a, z}$ X. C. Zhang, ${ }^{a}$ X. D. Hui, ${ }^{a}$ B. C. Wei, ${ }^{b}$ and G. L. Chen ${ }^{a}$ \\ ${ }^{a}$ State Key Laboratory for Advanced Metals and Materials, University of Science and Technology \\ Beijing, Beijing 100083, China \\ ${ }^{b}$ National Microgravity Laboratory, Institute of Mechanics, Chinese Academy of Sciences, Beijing 100080 , \\ China
}

\begin{abstract}
Porous $\mathrm{Zr}$-based bulk metallic glass (PMG) with unidirectional opening pores is prepared by electrochemical etching of tungsten wires of the W/bulk metailic glass (BMG) composites. The porosity and pore size can be controlled by adjusting the tungsten wires. The PMG showed no measurable loss in thermal stability as compared to the monolithic Zr-based BMG by water quenching and is more ductile and softer than the pore-free counterpart. The specific surface area of the PMGs is calculated to be $0.65,3.96$, and $10.54 \mathrm{~m}^{2} / \mathrm{kg}$ for 20,60 , and $80 \mathrm{vol} \%$ porosity, respectively.

(C) 2007 The Electrochemica] Society. [DOI: 10.1149/1.2787868] All rights reserved.
\end{abstract}

Manuscript submitted July 3, 2007; revised manuscript received August 17, 2007. Available electronically October 8, 2007.

Porous metallic glass (PMG) materials have received increasing interest in recent years because they combine the advantages of netallic foams and metallic glasses. ${ }^{1-8}$ Metallic foam materials exhibit properties like high specific strength and high energyabsorption capacity, ${ }^{9}$ whereas metallic glasses have distinctive characteristics like high strength, wear resistance, and strong corrosion 'resistance combined with desirable properties at relatively low densities. ${ }^{10}$ Thus, PMGs may find possible applications as fluid filiers, catalytic substrates, and biomedical implants. ",2

Most of the processing technique for PMG is a replication or reformation method used previously in the production of crystalline metal foams, ${ }^{1-8}$ and they can be divided into two groups: liquid state/supercooled liquid state processing and solid-state processing. For the first case, pores of metallic glasses were made under liquid state and supercooled liquid state with the aid of some pore-formers, such as gas-generating flux additives, ${ }^{3}$ soluble place holders, ${ }^{4,5}$ and hollow carbonspheres. ${ }^{6}$ These techniques all require that the metallic glass systems have a high glass-forming ability to allow synthesis of the final PMG, as the introduction of pores during foaming decreases thermal conductivity of the melt. Some new porous forming techniques for metallic glass foaming by means of solid-state processing have also been developed. For example, a porous Ti-based metallic glass has been fabricated by applying the principle of selective phase dissolution in $\mathrm{Y}_{20} \mathrm{Ti}_{36} \mathrm{Al}_{24} \mathrm{Co}_{20}$ two-phase amorphous alloy, ${ }^{7}$ and Lee et al. synthesized uniformly distributed nanoscale pores within a $\mathrm{Cu}_{47} \mathrm{Ti}_{33} \mathrm{Zr}_{11} \mathrm{Ni}_{8} \mathrm{Si}_{1}$ meiallic glass by extruding layered $\mathrm{Cu}$-based metallic glass + $\mathrm{Cu}$ composite powders following dissolution of the $\mathrm{Cu}$ in a $\mathrm{HNO}_{3}$ solution.

Until now, the PMGs prepared by the above-mentioned methods have contained a disordered pore structure. Materials with unidirectional opening pores may have some special potential application in the fields of engineering, electronics, photonics, and life sciences. ${ }^{11}$ For porous crystalline metals, Higuchi et al. reported the biocompatibility of lotus type porous stainless and titanium in alveolar bone in order to investigate the possibility of an implant by animal experiments, then concluded that titanium can be utilized to create pores of $150-200 \mu \mathrm{m}$ in diameter which would allow growth of bony tissues alone in the pores to enhance holding force. ${ }^{12}$ In the current study, we report the fabrication of porous $\mathrm{Zr}_{47} \mathrm{Ti}_{13} \mathrm{Cu}_{11} \mathrm{Ni}_{10} \mathrm{Be}_{16} \mathrm{Nb}_{3}$ bulk metallic glasses (BMGs) with uniformly dispersed unidirectional pores by sequential processes. The alloy is oniy a model one which can be changed to some other commercial BMG alloy, like Vit106 $\left(\mathrm{Zr}_{57} \mathrm{Nb}_{5} \mathrm{Cu}_{15.4} \mathrm{Ni}_{12.6} \mathrm{Al}_{10}\right)$ or $\mathrm{Cu}_{47} \mathrm{Ti}_{33} \mathrm{Zr}_{11} \mathrm{Ni}_{8} \mathrm{Si}_{1}$ BMG alloy.

$\mathrm{Zr}$-based alloy ingots were prepared by arc melting a mixture of pure metal elements (purity $99.9 \%$ or greater) in a titanium-gettered argon atmosphere. Unidirectional tungsten wires with a diameter of about $250 \mu \mathrm{m}$ were straightened and cut to $5 \mathrm{~cm}$ in length, then degreased by ultrasonic cleaning in a bath of acetone followed by the same procedure in a bath of ethanol. The tungsten/BMG composite samples containing 20,60, and 80 vol \% tungsten wires by volume fraction were prepared by melt infiltration casting. ${ }^{13}$ The tungsten wires were placed in a quartz tube with an inner diameter of $4 \mathrm{~mm}$ and then molten master alloy ingot was infiltrated among them by applying gas pressure. The tube was then quickly removed from the furnace and quenched in a brine solution, and the tungsten/ BMG composite samples having uniformly dispersed tungsten wires were prepared. For comparison, monolithic Zr-based BMG was prepared by water quenching. Then the tungsten/BMG composite samples were cut to a length of $7 \mathrm{~mm}$ and etched by the electrochemistry method in a sodium hydroxide electrciyte (5-10 wt \%) operated at voltage of $12 \mathrm{~V}$. The composite specimens were clamped on a stainless steel specimen holder and two-thirds of the samples were vertically immerged in the electrolyte, and the samples worked as anodic electrode, while a stainless steel plate worked as cathodal electrode. After some time, the tungsten wires in the composite samples were removed and honeycomb porous bulk metallic glass was obtained.

In the case of sodium hydroxide electrolyte, because of the high corrosion resistance of $\mathrm{BMG}$, the cathodal and anodic reactions are as follows

Cathode

$$
6 \mathrm{H}_{2} \mathrm{O}+6 \mathrm{e}^{-} \rightarrow 3 \mathrm{H}_{2}+6 \mathrm{OH}^{-}
$$

Anode

$$
\mathrm{W}+8 \mathrm{OH}^{-} \rightarrow \mathrm{WO}_{4}^{2-}+4 \mathrm{H}_{2} \mathrm{O}+6 \mathrm{e}
$$

Combining Eq. 1 and 2, we get the following formula

$$
\mathrm{W}+2 \mathrm{OH}^{-}+2 \mathrm{H}_{2} \mathrm{O} \rightarrow \mathrm{WO}_{4}^{2-}+3 \mathrm{H}_{2}
$$

The hydrogen gas produced from the cathodal reaction carries the electrolyte upward along with the pores, which are formed during the dissolution of the tungsten wires. This makes the sample in the electrolyte react continuously, and finally the tungsten wires are etched completely. Density is measured by the Archimedian principle. The density of monolithic Zr-based BMG is $6.11 \mathrm{~g} / \mathrm{cm}^{3}$.

$\mathrm{X}$-ray diffraction (XRD) analyses of the samples were performed using $\mathrm{Cu} K \alpha$ radiation (Rigaku $D_{\max }-\mathrm{RB}$ ). Differential scanning calorimetry (DSC) was performed with a Netzsch DSC 404C differential scanning calorimeter under argon atmosphere. A constant heating rate of $20 \mathrm{~K} / \mathrm{min}$ was employed. A Zeiss Supra 55 scanning electron microscope was used to examine the cross-sectional morphologies of the porous bulk metallic glasses. Compressive testing was performed under displacement control at a constant displacement rate of $5 \times 10^{-4} \mathrm{~s}^{-1}$ on a CMT4305 mechanical testing apparatus. 

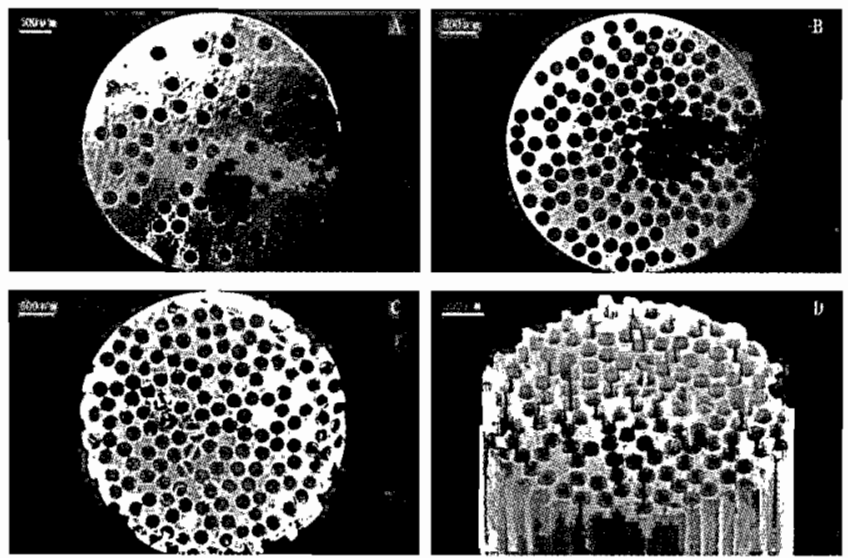

Figure 1. SEM images of the cross sections of porous Zr-based BMGs with nominal porosities of (A) 20, (B) $60^{\circ}$, and (C) $80 \mathrm{vol} \%$, respectively. A side view of $(\mathrm{C})$ is shown in (D), illustrating the unidirectional pore structure.

Scanning electron microscopy (SEM) images of the porous $\mathrm{Zr}$ based BMGs with nominal 20,60 , and $80 \mathrm{vol} \%$ porosities are shown in Fig. 1. The pores in the PMGs are macroscopically homogeneous and honeycomb-shaped (Fig. IA-1C) and the size of the individual pore is about $250 \mu \mathrm{m}$ in diameter. A side view of Fig. IC is shown in Fig. 1D, illustrating the unidirectional pore structure.

The amorphous structure of the porous $\mathrm{Zr}$-based BMG was identified by XRD. The XRD pattern taken from the cross section of the PMG with 60 vol \% porosity is shown in Fig. 2. For comparison, XRD patterns taken from the W/Zr-based BMG composite precursor and tungsten wires are also included. Crystalline peaks corresponding to elemental $\mathrm{W}$ are presented in the precursor sample, and only a broad diffraction maximum, characteristic of amorphous structure, is observed in the PMG. These results indicate that this PMG is composed of a single amorphous phase. The PMGs with 20 and $80 \%$ porosities show the same characteristics.

The DSC curve of the porous Zr-based BMG with $60 \mathrm{vol} \%$ porosity at the heating rate of $20 \mathrm{~K} / \mathrm{min}$ under argon is shown in Fig. 3, together with the data of the monolithic $\mathrm{Zr}$-based (pore-free) sample prepared by water quenching. The thermogram of the porous alloy exhibits a glass transition endotherm (onset at $T_{\mathrm{g}}$ ) and a crys-

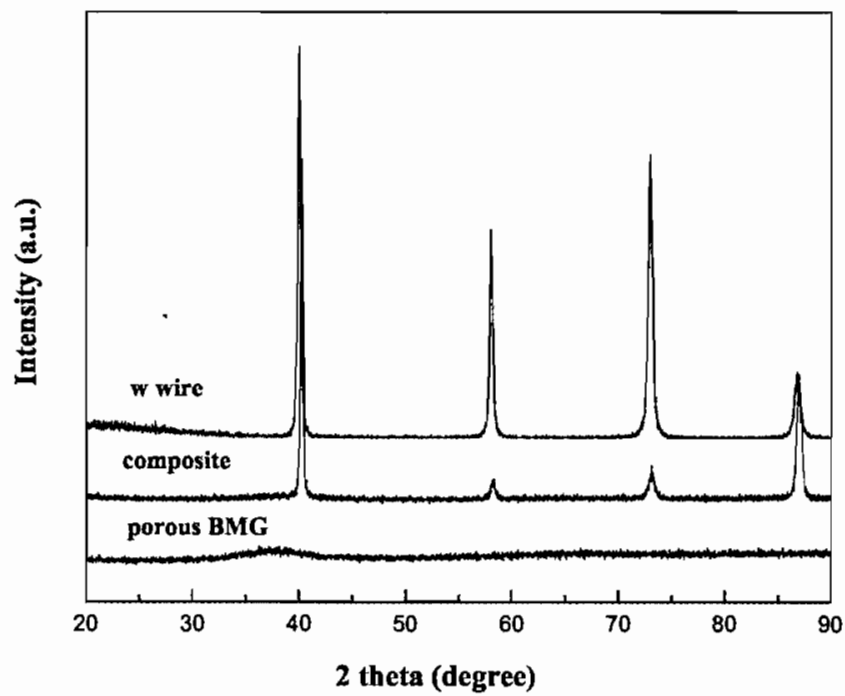

Figure 2. XRD pattern taken from the cross section of the porous $\mathrm{Zr}$-based BMG with porosity of $60 \mathrm{vol} \%$. For comparison, XRD patterns taken from the W/Zr-based BMG composite precursor and tungsten wires are included.

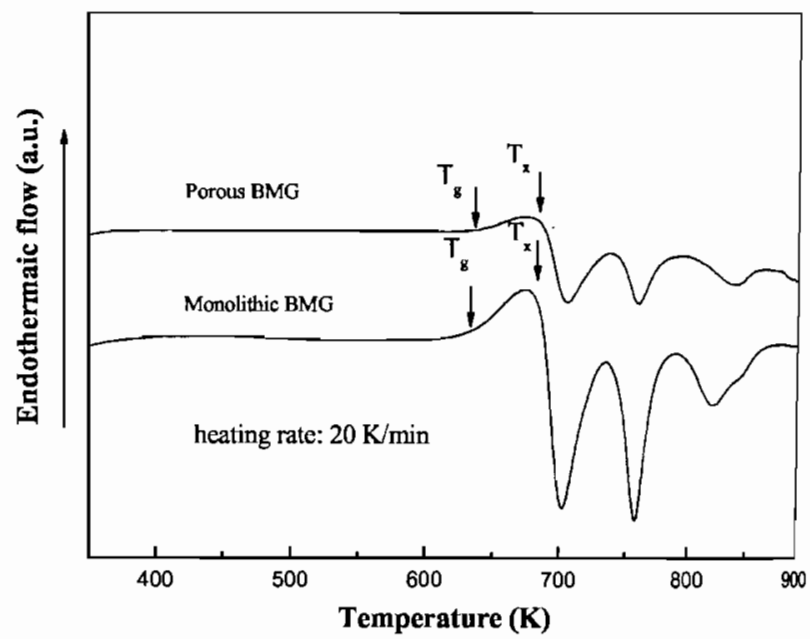

Figure 3. DSC trace of the porous Zr-based BMG with porosity of $60 \mathrm{vol} \%$, showing a glass transition at $638 \mathrm{~K}$ followed by crystallization. The data of the monolithic $\mathrm{Zr}$-based BMG prepared by water quenching is shown for comparison.

tallization exotherm (onset at $T_{x}$ ) appearing at 638 and $688 \mathrm{~K}$, respectively, in agreement with those of the monolithic sample.

The typical compressive engineering stress-strain curve of the honeycomb porous $\mathrm{Zr}$-based BMG with the porosity of $60 \mathrm{vol} \%$ is shown in Fig. 4, together with the data of the pore-free alloy prepared by water quenching. The porous alloy is more ductile and softer than the pore-free counterpart. The yield strength $\left(v_{0.2}\right)$ is about 820 and $2000 \mathrm{MPa}$ for the porous and pore-free BMG, respectively. The corresponding yield strain is 1.3 and $1.7 \%$, respectively. The porous sample exhibits a plastic strain of $0.6 \%$ before failure, while the value of the pore-free sample is only $0.3 \%$. A SEM mcrograph depicting the shear bands forming and then arrested by the fibrous channel during compressive deformation for the porous $\mathrm{Zr}$ based BMG with a porosity of $60 \mathrm{vol} \%$ is shown in Fig. 5. Brothers pointed out that pores in porous metallic glass may confine shearband propagation and increase shear-band densities by arresing the small number of bands normally responsible for compressive failure in monolithic bulk metallic-glass samples, in much the same way that reinforcing metallic or ceramic phases enhance compressive ductility in amorphous metal-matrix composites, and that improvements in ductility through introduction of low $(<70 \%)$ porosity

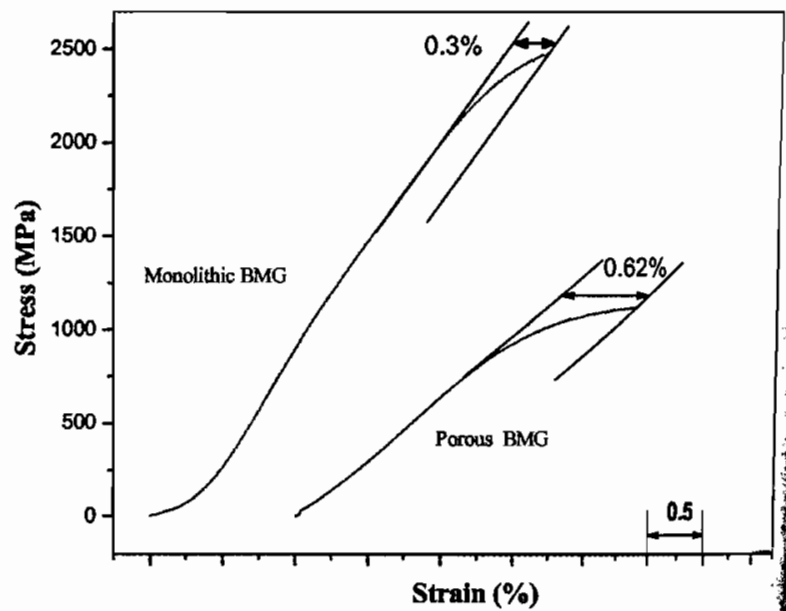

Figure 4. Compressive stress-strain behavior of the porous Zr-based But with porosity of $60 \mathrm{vol} \%$. The data of the monolithic Zr-based BMG p. pared by water quenching is shown for comparison. 


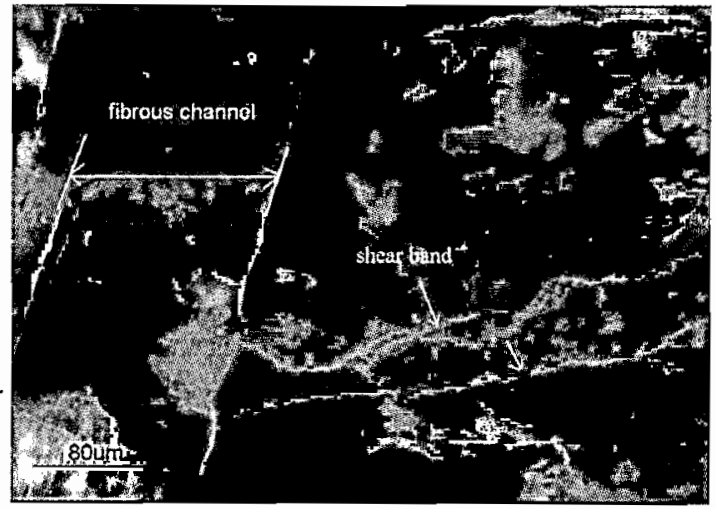

Figure 5. SEM micrograph depicting the shear bands forming and then arrested by the fibrous channel during compressive deformation for the porous Zr-based BMG with porosity of $60 \mathrm{vol} \%$.

levels are probably best rationalized in terms of unstable shear-band arrest. 'So, the fibrous channel in Fig. 5 confines single shear-band propagation by arresting the small number of shear bands and thus allows the formation of multiple shear bands in the PMG, which promotes stable crack growth and extends the PMG strain to failure.

Using the BMG density data, the specific surface area of the present honeycomb porous BMGs is calculated to be $0.65,3.96$, and $10.54 \times 10^{-3} \mathrm{~m}^{2} / \mathrm{g}$ for 20,60 , and $80 \mathrm{vol} \%$ porosity, respectively. The whole density of the PMG can be calculated by the following equation

$$
\rho_{\mathrm{PMG}}=\rho_{\mathrm{BMG}}\left(1-V_{\mathrm{p}}\right)
$$

Here, $\rho_{\mathrm{PMG}}$ and $\rho_{\mathrm{BMG}}$ are the whole density of the porous BMG and the monolithic $\mathrm{BMG}$ and $V_{\mathrm{p}}$ is the volume fraction of the porosity. According to Eq. 4, the whole density of the PMG with $80 \mathrm{vol} \%$ porosity is about $1.2 \mathrm{~g} / \mathrm{cm}^{3}$, which is a very lightweight material. Furthermore, the size and distribution of pores are controllable by adjusting the diameter and volume fraction of the tungsten wires during melt infiltration. Also, the composite precursor can be selected flexibly; for example, the tungsten wires can be replaced by copper wires. ${ }^{14}$

\section{Acknowledgments}

The authors thank Professor Zidong Wang, Dr. Meiling Wang, and Dr. C. T. Liu for helpful discussions. Financial support from the National Natural Science Foundation of China (grant no. 50571018), the Program for New Century Excellent Talents in University (NCET), and the Program of Introducing Talents of Discipline to Universities, project no. B07003 are greatly acknowledged.

University of Science and Technology Beijing assisted in meeting the publication costs of this article.

\section{References}

1. A. H. Brothers, Scr. Mater, 54, 513 (2006).

2. S. Hiromoto, K. Asami, A. P. Tsai, and T. Hanawa, Mater. Trans., 43, 261 (2002).

3. J. Schroers, C. Veazey, and W. L. Johnson, Appl. Phys. Lett., 82, 370 (2003).

4. T. Wada and A. Inoue, Mater. Trans., 44, 2228 (2003).

5. A. H. Brothers and D. C. Dunand, Adv. Mater. (Weinheim, Ger.), 17, 484 (2005).

6. A. H. Brothers and D. C. Dunand, Appl. Phys. Lett., 84, 1108 (2004).

7. J. Jayaraj, B. J. Park, D. H. Kim, W. T. Kim, and E. Fleury, Scr. Mater, 55, 1063 (2006).

8. M. H. Lee and D. J. Sordelet, Scr. Mater, 55, 947 (2006).

9. J. Banhart, Prog. Mater. Sci., 46, 559 (2001).

10. A. Inoue, Acta Mater., 48, 279 (2000).

11. M. L. Kai Hoa, M. Lu, and Y. Zhang, Adv. Colloid Interface Sci., 121, 9 (2006).

12. H. Nakajima, Prog. Mater. Sci., 52, 1091 (2007).

13. R. B. Dandliker, R. D. Conner, and W. L. Johnson, J. Mater. Res., 13, 2896 (1998).

14. P. Wadhwa, J. Heinrich, and R. Busch, Scr. Mater, 56, 73 (2007). 